INPLASY

PROTOCOL

To cite: Yi et al. Effectiveness of Banxia Xiexin Decoction in the treatment of precancerous lesions: A protocol for systematic review and metaanalysis. Inplasy protocol

202130102. doi:

10.37766/inplasy2021.3.0102

Received: 27 March 2021

Published: 28 March 2021

Corresponding author:

Ziyi Hu

huziyi0829@163.com

Author Affiliation:

The Affiliated Hospital of

Jiangxi University of

Traditional Chinese Medicine

Support: Grant No.2020.2.

Review Stage at time of this submission: The review has not yet started.

Conflicts of interest:

None declared.

\section{Effectiveness of Banxia Xiexin Decoction in the treatment of precancerous lesions: A protocol for systematic review and meta-analysis}

Yi, Y1; Hu, ZY²; Li, RL³; Chen, LS4; Zhang, HY5; Li, H6; $\mathrm{Wu}, \mathrm{MW}^{7}$; Liu WT8.

Review question / Objective: 1.Type of studies. This study will include all relevant randomized clinical trials (RCTs) without language restrictions. 2.Type of participants. Patients with chronic atrophic gastritis or with intestinal metaplasia or dysplasia diagnosed by gastroscopy, pathological examination and laboratory examination have clinical manifestations such as long-term dyspepsia, anorexia, fatigue, epigastric distension, emaciation, anemia and so on. Positive for $\mathrm{Hp}$. The patients were regardless of age, sex or race. 3.Type of interventions. BXD is used in the treatment of precancerous lesions of gastric cancer in the form of decoction, powder, capsule and so on. 4.Type of comparators. Oral routine western medicine with variable dosage form. 5.Types of outcome measures. The total effective rate of clinical remission, the effective rate of gastroscopy, the clearance rate of $\mathrm{Hp}$ and the effective rate of pathological examination.

INPLASY registration number: This protocol was registered with the International Platform of Registered Systematic Review and Meta-Analysis Protocols (INPLASY) on 28 March 2021 and was last updated on 28 March 2021 (registration number INPLASY202130102).

\section{INTRODUCTION}

Review question / Objective: 1.Type of studies. This study will include all relevant randomized clinical trials (RCTs) without language restrictions. 2. Type of participants. Patients with chronic atrophic gastritis or with intestinal metaplasia or dysplasia diagnosed by gastroscopy, pathological examination and laboratory examination have clinical manifestations such as long-term dyspepsia, anorexia, fatigue, epigastric distension, emaciation, anemia and so on. Positive for $\mathrm{Hp}$. The patients were regardless of age, sex or race. 3.Type of interventions. BXD is used 
in the treatment of precancerous lesions of gastric cancer in the form of decoction, powder, capsule and so on. 4.Type of comparators. Oral routine western medicine with variable dosage form. 5.Types of outcome measures. The total effective rate of clinical remission, the effective rate of gastroscopy, the clearance rate of $\mathrm{Hp}$ and the effective rate of pathological examination.

Condition being studied: Gastric precancerous lesion(GPL) is a necessary stage in the occurrence and development of gastric cancer. At present, the incidence of gastric cancer is increasing year by year. It's important to prevent and control gastric cancer through early detection and intervention. Banxia Xiexin decoction(BXD) has a good effect in improving symptoms, reducing inflammation, promoting the repair of gastric mucosa, reversing its atrophy and intestinal metaplasia.BXD may be a foreground choice for the treatment of GPL.

\section{METHODS}

Participant or population: Patients with chronic atrophic gastritis or with intestinal metaplasia or dysplasia diagnosed by gastroscopy, pathological examination and laboratory examination have clinical manifestations such as long-term dyspepsia, anorexia, fatigue, epigastric distension, emaciation, anemia and so on. Positive for $\mathrm{Hp}$. The patients were regardless of age, sex or race.

Intervention: BXD is used in the treatment of precancerous lesions of gastric cancer in the form of decoction, powder, capsule and so on.

Comparator: Oral routine western medicine with variable dosage form.

Study designs to be included: This study will include all relevant randomized clinical trials (RCTs) without language restrictions.

Eligibility criteria: 1.Type of studies. This study will include all relevant randomized clinical trials (RCTs) without language restrictions. 2.Type of participants.Patients with chronic atrophic gastritis or with intestinal metaplasia or dysplasia diagnosed by gastroscopy, pathological examination and laboratory examination have clinical manifestations such as longterm dyspepsia, anorexia, fatigue, epigastric distension, emaciation, anemia and so on. Positive for $\mathrm{Hp}$. The patients were regardless of age, sex or race. 3.Type of interventions. BXD is used in the treatment of precancerous lesions of gastric cancer in the form of decoction, powder, capsule and so on. 4.Type of comparators. Oral routine western medicine with variable dosage form. 5.Types of outcome measures. The total effective rate of clinical remission, the effective rate of gastroscopy, the clearance rate of $\mathrm{Hp}$ and the effective rate of pathological examination.

Information sources: RCTs of BXD for gastric precancerous lesions will be searched in the relevant database, including PubMed, Embase, Cochrane Library, China National Knowledge Infrastructure (CNKI), Wanfang Database, Chinese Biomedical Literature Database (CBM) and Chinese Scientific Journal Database (VIP database). The studies of electronic searches will be exported to EndNote V.9.1 software. We will run metaanalyses using the Review Manager (RevMan) V.5.3 software. Any disagreement will be solved in consultation with a third reviewer.

Main outcome(s): The total effective rate of clinical remission, the effective rate of gastroscopy, the clearance rate of $\mathrm{Hp}$ and the effective rate of pathological examination.

Data management: We will establish a data extraction table, which will be used by 2 researchers to extract data from qualified literature. The specific contents will include: author, publication time, participant characteristics, intervention (s), comparison (s), outcome (s), adverse events and some relevant features. If the significant information of the article is incomplete, we will contact the author. In 
case of disagreements, the third researcher will be consulted.

Quality assessment / Risk of bias analysis: The Cochrane risk assessment tool will be used by us to evaluate the methodological quality of qualified RCTs. It includes 7 items: random sequence generation, allocation concealment, blinding of participants and caregivers, blinding of outcome assessors, incomplete outcome data, selective outcome reporting, and other bias. The evaluation result of each item will be "high risk", "low risk", or "unclear risk". The assessment will be completed by 2 reviewers, and disagreements will be handed over to the third reviewer for the final decision.

Strategy of data synthesis: Data synthesis will be completed using RevMan5.3.5 software. When $12<50 \%$, we will choose the fixed effects model; Otherwise, the random effects model will be selected. The forest plots will present the results of the metaanalyses. We will conduct descriptive analysis, when the results are not suitable for consolidation. When more than 10 studies are included, we will use the funnel plot to assess publication bias.

Subgroup analysis: If necessary, subgroup analyses will be performed according to the different types of participant characteristics, treatment methods, treatment frequency, and so on.

Sensitivity analysis: When there is significant heterogeneity, we will conduct a sensitivity analysis. We will determine the robustness of the results by excluding lowquality studies.

Country(ies) involved: China.

Keywords: Chronic atrophic gastritis; gastric precancerous lesions; Banxia Xiexin Decoction; protocol.

Contributions of each author:

Author 1 - Ying Yi.

Email: 1214986471@qq.com

Author 2 - Ziyi Hu.

Email: huziyi0829@163.com
Author 3 - Renliang Li.

Email: 1051545802@qq.com

Author 4 - Lisha Chen.

Email: 1071342707@qq.com

Author 5 - Hengyi Zhang.

Email: 814520785@qq.com

Author 6 - Hong Li.

Email: 546872508@qq.com

Author 7 - Mengwen Wu.

Email: 1225661717@qq.com

Author 8 - Wanting Liu.

Email: 393288786@qq.com 